\title{
Gonadal suppression alters axillary steroid secretions in men, but does that affect olfactory social signaling?
}

\author{
Syed Imran Ali Shah ${ }^{1, *}$, Hannah C. P. Wilson ${ }^{1}$, Paul D. Abel ${ }^{1}$, Richard J. Wassersug ${ }^{2, *}$, \\ Emma S. Tomlinson Guns ${ }^{7}$, S. Craig Roberts ${ }^{8}$ \\ ${ }^{1}$ Department of Surgery \& Cancer, Imperial College London, SW7 1 London, UK \\ ${ }^{2}$ Department of Cellular \& Physiological Sciences, University of British Columbia, Vancouver, BC V6T, Canada \\ ${ }^{3}$ National Institute of Mental Health, 25067 Klecany, Czech Republic \\ ${ }^{4}$ Faculty of Physical Education and Sport, Charles University, 12000 Prague, Czech Republic \\ ${ }^{5}$ Faculty of Science, Charles University, 12000 Prague, Czech Republic \\ ${ }^{6}$ School of Psychology, Newcastle University, NE2 4 England, UK \\ ${ }^{7}$ Vancouver Prostate Centre, Vancouver, BC V4C, Canada \\ ${ }^{8}$ Division of Psychology, University of Stirling, FK9 4 Scotland, UK
} Vít Třebický ${ }^{3,4}$, Jitka Třebická Fialová ${ }^{3,5}$, Caroline Allen ${ }^{6}$, Hans H. Adomat ${ }^{7}$, Robert H. Bell ${ }^{7}$,

*Correspondence: s.shah10@alumni.imperial.ac.uk (Syed Imran Ali Shah); richard.wassersug@ubc.ca (Richard J. Wassersug)

\begin{abstract}
Background and objective: Luteinizing hormone-releasing hormone agonists (LHRHa) suppress gonadal hormone production and are commonly used to treat prostate cancer (PC) in men and conditions ranging from uterine fibroids to estrogen-sensitive cancers in women. They are also used to delay sexual development in children considering gender reassignment or experiencing premature puberty. As chemically castrating agents, LHRHa may affect cutaneous steroid secretions, which, in turn, could alter body odor and influence the psycho-sexual dynamics between individuals. The objectives of the present study were to determine (1) if LHRHa indeed alter cutaneous skin secretions, and (2) whether this leads to perceivable changes in body odor.

Material and methods: Axillary skin secretions were collected on new cotton T-shirts worn by men undergoing androgen deprivation therapy with an LHRHa to treat PC $(n=10)$, both before starting the LHRHa and 3 months later. Healthy heterosexual university students (50 males, 50 females) were recruited to smell and rate the shirts for their masculinity, attractiveness, and intensity of odor. Liquid chromatography-mass spectrometry (LC-MS) was also used to analyze steroids extracted from the shirt samples.

Results: LC-MS showed a statistically significant decline in the concentration of the androgenic metabolites, androsterone and $5 \alpha$-androstane-3,17-dione. This confirms that LHRHa drugs that suppress gonadal hormone production markedly reduce cutaneous secretion of androgenic metabolic intermediates in adult males. However, no differences in odor were detected in the ratings of the shirts by male, female, nor male and female raters combined for any of the three variables assessed. Possible reasons why the human sniffers failed to perceive a change in odor are explored.

Conclusion: Our data document that LHRHa alter steroid skin secretions in older men, but whether such changes alter the olfactory signals that might influence psychosocial interactions remains unresolved.
\end{abstract}

\section{Keywords}

Prostate cancer; Androgen deprivation therapy; Scent; Olfaction; Social implications

\section{Introduction}

Luteinizing hormone-releasing hormone agonists (LHRHa) are a class of synthetic hormones that reduce endogenous go- nadal hormone production in both men and women through the modulation of the hypothalamic-pituitary-gonadal axis. LHRHa induced continuous, non-physiological stimulation of the pituitary gland eventually leads to downregulation of 
pituitary luteinizing hormone-releasing hormone (LHRH) receptors. Through a negative feedback mechanism, this causes subsequent reduction in luteinizing hormone (LH) production and gonadal hormone synthesis [1]. LHRHa are thus used clinically to treat gonadal hormone-sensitive medical conditions. For men, they are the most common agent used for androgen deprivation therapy (ADT) in the treatment of prostate cancer (PC) [2]. In women they are used to treat a variety of conditions such as uterine fibroids, endometriosis, and gonadal hormone-sensitive cancers. They are also used to delay sexual development in children considering gender-affirming therapy (GAT) or experiencing premature puberty [3]

We explore here the impact of LHRHa administration on steroid skin secretions focusing on men with PC receiving LHRHa for ADT. LHRHa reduce serum testosterone (T) levels by $>90 \%$ in men. Since estradiol (E2) in males is derived directly from $\mathrm{T}$ by the enzyme aromatase, LHRHa also reduce the E2 levels by some $80 \%$ [4]. This iatrogenic hypogonadism leads to complications, some of which, like erectile dysfunction, loss of libido, and decreased muscle mass, are attributable to low T. Others, such as osteoporosis and hot flashes, are primarily due to E2 deficiency [5, 6].

ADT's adverse effects on men's sexual function reduce patients' masculine self-esteem and quality of life (QoL) $[7,8]$. $\mathrm{T}$ is a social hormone [9] and, as such, its impact on men indirectly affects their intimate partners [10]. The partners of patients on ADT may in fact be more distressed than the patients themselves $[11,12]$. A small $(n=15)$ interview based study of men receiving ADT revealed that nearly half experienced erosion of spousal relations, which was not limited to intimate sexual contact, but also affected other aspects of their social relationships [13].

In most mammals, olfaction is the dominant sensory modality in social communication [14, 15]. Humans possess the ability to perceive socially relevant information from chemical signaling, which varies from individual to individual [16]. Different areas of the human body have characteristic odors. Of these, axillary scent is most pungent and generally considered the primary source of body odor. Axillary odor is affected by an individual's genetics, ethnicity, sex, personality, sexual orientation, diet and health $[17,18]$. Exposure to axillary apocrine secretion from conspecific individuals has been shown to elicit psychological and physiological responses [19]. It is thus possible that relationship changes for patients on LHRHa and their partners may be affected by changes in the chemical signal that patients emit.

Perception of a male's scent contributes to women's assessments of partners, providing cues about a potential partner's health, reproductive status, and genetic quality [20]. Perceived pleasantness of male axillary scent is also positively associated with measures of male masculinity and dominance [21], as well as underlying T levels [22]. The axillary chemical signals from men may change with age [23], but it is not yet known whether this is due to age-related variations in their sex hormone profile. It is in fact not known whether
LHRHa significantly alters cutaneous axillary secretions in PC patients or other populations treated with gonadal hormone suppressing drugs. Similarly, it is not known whether any such changes influence the olfactory signals patients emit in a way that might impact on their intimate relationships.

Axillary apocrine secretions contain various odoriferous volatile chemicals [20]. Some, such as the androstenes, are intermediate products in the metabolic conversion of cholesterol to the primary sex hormones and are variously aromatic [14, 24]. Androstenes have been shown to act as chemosignals that can influence human behavior in multiple ways such as sexual interest, attraction, mood, emotions and possibly mate selection [25-29]. These observations raise the question of whether some component of the erosion of spousal relations for PC patients on LHRHa drugs may be the result of changes in the olfactory signal emitted by androgen suppressed patients.

In this study we address two questions. First, does ADT achieved with LHRHa change the excreted cutaneous steroids from the axillary region of PC patients compared to baseline? We also ask whether there is any perceivable difference in the axillary scent of men on ADT compared again to baseline and to age-matched controls with normal levels of gonadal hormones.

\section{Methods}

\subsection{General sample collection}

We collected samples of axillary secreted steroids absorbed on T-shirts worn by ten participants following an established protocol used previously in investigations of axillary scent [30-32].

A longitudinal design was employed. Study samples were provided by men between 50-75 years old from the urooncology clinics of Imperial College Healthcare NHS Trust. These men were all being treated for PC and about to begin ADT with an LHRHa drug (monthly subcutaneous Goserelin implant). The patients provided samples of axillary sweat and blood for serum $\mathrm{T}$ and E2 at baseline before starting hormone treatment and at three months after initiation of ADT. The patients received an anti-androgen (oral bicalutamide) one week before the administration of LHRHa as per the routine clinical management to prevent the testosterone flare-up phenomenon. The patients did not have any history of the use of inhibitors of androgen biosynthetic enzyme cytochrome P450 17 $\alpha$ hydroxylase/17, 20 lyase (CYP17), steroidal medications or any other anti-cancer drug. An additional 10 age-matched men, without a diagnosis of PC, served as controls. The control participants also did not have any history of use of any steroidal pharmacologic agent. Their samples of axillary sweat and blood were also taken twice with a three-month interval. All samples were collected after approval as an Imperial College Healthcare Tissue Bank (ICHTB) sub-collection and were later issued for analysis following ICHTB ethics approval.

Materials provided to the study participants for axillary sample collection included the T-shirt (prewashed in non- 
perfumed detergent), a zip-lock plastic storage bag for the shirt, and a non-perfumed soap bar (Simple Pure soap bar, UK). Participants were required to wear the T-shirt overnight for two consecutive nights. They were asked to follow a set of instructions commencing 24 hours before sampling. This included abstinence from sexual activity, sleeping alone, avoiding deodorants and other perfumed products, wearing only the provided $\mathrm{T}$-shirt while sleeping, avoiding tobacco smoke, alcohol and strong-smelling foods (e.g., chilli, garlic, pepperoni, curry, blue cheese, asparagus, yogurt and fried onion).

Subjects were required to shower just before retiring alone to bed on each of the two consecutive nights using only the soap provided, wearing the $\mathrm{T}$-shirts in bed and returning them to the storage bag each morning. T-shirts were collected and stored at $-80{ }^{\circ} \mathrm{C}$ on the day after the second night. Before long term storage, the bottom half of each T-shirt, below the rib cage, was discarded and the remaining part was cut into right and left halves which were stored separately in sealable plastic bags labelled in an anonymized form with key codes indicating only the group (PC or control) and the time point of sampling (baseline or 3 months).

Quantitative determination of serum $\mathrm{T}$ and E2 in venous blood samples from participants was carried out at Imperial Clinical Chemistry Laboratory, Charing Cross Hospital, London using ARCHITECT ${ }^{\circledR}$ assays (Abbott Laboratories, Abbott Park, Illinois, USA).

\subsection{Olfactory assessment}

The right halves were transported to the University of Stirling, Scotland, and immediately put into a $-20{ }^{\circ} \mathrm{C}$ freezer. The next morning, the plastic bags containing the $\mathrm{T}$-shirt samples were removed from the freezer and allowed to thaw for 2 hours at room temperature. Each bag was shaken thoroughly and inverted 3-4 times to uniformly distribute volatile compounds.

A group of 100 healthy heterosexual university students (50 women, 50 men, 17-34 years old with a mean age of 21.4 years) were recruited (after provision of study information and receiving written informed consent as approved by the University of Stirling Research Ethics Committee) to smell and rate individual samples for their masculinity, attractiveness, and intensity. Only women using hormonal contraception were recruited to rate the smell of the samples so as to avoid the potential effect of fluctuations in olfactory function during the natural menstrual cycle [33]. Participants rated samples on a 7-point scale (from 1-very low to 7-very high) and were also asked to complete a questionnaire on their basic demographic data (age, sex) and contraceptive history. Each participant on the olfactory assessment panel then smelled and rated a randomly allocated series of $8 \mathrm{~T}$ shirt samples (baseline and 3-month samples from 2 controls and 2 PC patients each). Raters were blind to the identity of the samples and their responses were kept anonymous.

\subsubsection{Statistical analysis}

Data were statistically analyzed in an anonymized form. For each individual at the two time points, mean scores were computed for attractiveness, masculinity and intensity ratings by male raters, female raters, and all raters combined. The rating averages and serum sex hormones data were analyzed using SPSS version 22.0 (SPSS Inc., Chicago, IL, USA). Means \pm standard error of mean (S.E.M) were calculated for normally distributed quantitative variables. Odor ratings were analyzed using repeated measures ANOVA, with odor sample (baseline, 3 month) as the within-subject factor and group (control, study) as the between-subject factor. If ADT influences men's scent, we would predict a significant sample $\mathrm{x}$ group interaction in these models. Pearson's correlation was applied to observe correlation between odor ratings and serum sex hormone levels. A $P$-value of $<0.05$ was considered statistically significant.

\subsection{Chemical assessment}

The left side of the frozen $\mathrm{T}$-shirts from the study group were shipped on dry ice to the Vancouver Prostate Centre in Canada. Included were two shirts that had not been worn and were in their original package as blank controls. All shirt samples were received while still on dry ice and immediately transferred to a $-80{ }^{\circ} \mathrm{C}$ freezer.

Sixteen steroids, all metabolic derivatives of cholesterol, were extracted from the shirt samples as described below. Liquid chromatography-mass spectrometry (LC-MS) was performed on the extracted samples using the protocol described previously [34, 35]. 10 samples were from men with PC before treatment and an equal number of samples were from the same men after three months on ADT. This yielded a final sample of 22 shirts, ten before ADT and ten three months after starting ADT, plus two unworn control shirts.

\subsubsection{Steroid extraction}

A swatch of T-shirt fabric approximately $200 \mathrm{mg}$ (weighed by differences) was placed into an $8 \mathrm{~mL}$ glass vial to which was added $5 \mathrm{~mL}$ ethyl acetate (EtOAc) and $20 \mu \mathrm{L}$ internal standard (IS, deuterated testosterone, d3T, and dihydrotesterone, d3DHT). Sample was rotated for 30 minutes and $4 \mathrm{~mL}$ extract transferred to a fresh vial. Extraction was repeated with an additional $4 \mathrm{~mL}$ EtOAc, pooled, and combined extracts dried under vacuum (Centrivap). Residue was taken up in $0.5 \mathrm{~mL}$ EtOAc and transferred to Eppendorf tubes, dried again, and $0.5 \mathrm{~mL} 100 \mathrm{mM}$ hydroxylamine/methanol (HA/MeOH) added and then well vortexed. The sample was next centrifuged (20000 g, $2 \mathrm{~min}$ ) and $45 \mu \mathrm{L}$ supernatant transferred to a fresh Eppendorf followed by addition of 45 $\mu \mathrm{L}$ water. The sample was again vortexed, centrifuged and the supernatant transferred to LC sample vials with inserts and heated at $60^{\circ} \mathrm{C}$ for 1 hour prior to LC-MS analysis. 


\subsubsection{LC-MS analysis}

The oxime derivatized steroids generated in the extraction protocol were analyzed using a Waters Acquity UPLC Separations Module coupled to a Waters Quattro Premier XE Mass Spectrometer. Separations were carried out with a 2.1 $\times 100 \mathrm{~mm}$ BEH $1.7 \mu \mathrm{M}$ C18 column, mobile phase water (A) and $0.1 \%$ formic acid in acetonitrile (B) (gradient: 0.2 min, 25\%B; 8 min, 70\%B; 9 min, 100\%B; 12 min 100\%B;12.2 min, 25\%B; 14 min run length). All data were collected in ES+ by multireaction monitoring with instrument parameters optimized for the $\mathrm{m} / \mathrm{z}$ 's and corresponding fragments of the oxime-steroids. Data processing was done with Quanlynx (Waters) and exported to Excel for additional normalization to weights and volumes as required. Final deuterated $\mathrm{T}$ and DHT levels in samples were 0.5 and $1 \mathrm{ng} / \mathrm{mL}$ respectively and a curve of 6 calibration standards $(0.01-10 \mathrm{ng} / \mathrm{mL})$ used for quantification $\left(\mathrm{R}^{2}>0.98\right)$. All ketosteroids in the androgen pathway were in the assay. Parameters were also optimized for cortisol and epi-testosterone were included in the acquisitions.

\subsubsection{Statistical analysis}

The mean values for each steroid in $\mathrm{ng} / \mathrm{mL}$ extracted from the shirts were assessed for normality with the Shapiro Wilks test. Only two of the 16 steroids had $P$-value greater than 0.1 indicative of a normal distribution. Normality did not improve when the data were log transformed. Thus, the data were considered non-parametric and compared with Wilcoxon rank sum test.

In order to assess correlations between shirts in one steroid concentration and another, the rank order of steroid concentrations was compared with Spearman's coefficient $\rho$. A similar analysis was done for each participant as well as the two unworn shirt samples as blank controls. The steroid concentrations before and after ADT were treated as ratios in the final statistical analysis.

\section{Results}

\subsection{Serum gonadal hormone levels}

As expected, $\mathrm{T}$ levels were significantly lower in the study group serum samples at 3 months than had been recorded at baseline (Serum T nmol/L, Mean \pm SEM; $16.56 \pm 1.45$ (baseline) vs. $0.64 \pm 0.12$ ( 3 months), $P<0.0001$ ). Similarly, E2 levels in the study group at 3-months assessment were significantly lower than baseline (Serum E2 pg/mL, Mean \pm SEM; $89.40 \pm 8.44$ (baseline) vs. $37.00 \pm 0.00$ ( 3 months), $P$ $<0.0001$ ). $37 \mathrm{pmol} / \mathrm{L}$ was the lowest detection limit of the E2 assay employed. No such change was observed over the three-month period in the T and E2 serum concentrations for control participants who were not treated with an LHRHa.

\subsection{Olfactory assessment}

No significant sample $\mathrm{x}$ group interactions were found in the ratings of either attractiveness, masculinity or intensity, whether the ANOVA model included assessments by male raters, female raters, or both male and female raters combined (Table 1).

\subsection{Chemical assessment}

The medians for the men in the study group at baseline and after three months of LHRHa administration along with the $P$ values for the Wilcoxon test comparison are presented in Table 2. These are in rank order from those with the greatest to least significant differences. With $\alpha$ set at 0.05 , only two of the seven androgenic steroids significantly differed in concentration between men before and after ADT. These were androsterone and $5 \alpha$-androstane-3,17-dione. Both were significantly lower (median drop after ADT was to $51 \%$ and $48 \%$ from the pre-treatment levels respectively) in the cutaneous secretion of the men after 3 months of ADT (Wilcoxon signed rank test; both $P=0.02$ ). Three more androgenic compounds had lower mean concentrations after three months of ADT, but not significantly so.

\section{Discussion}

Three months of ADT with LHRHa drugs alters the cutaneous steroid secretions in the axillary region of men when assessed with LC-MS. The shift is overall characterized by suppression of androgenic intermediates. Although ADT achieved with an LHRHa reduces gonadal steroid levels, production of mineralocorticoids and glucocorticoids is not suppressed indicating that their progestogen precursors are preserved. That is consistent with the presence of various progestogens in our cutaneous samples.

The suppression of the weaker androgens, androsterone and $5 \alpha$-androstane-3,17-dione, in the samples after ADT treatment is interesting in its own regard. These are the intermediate metabolites between the progestogen 5-pregnan17-ol-3,20-dione and T. The lack of gonadal conversion of progestogens to androgens is consistent with the drop in the concentration of the weaker androgens, androsterone and $5 \alpha$-androstane-3,17-dione, in the cutaneous axillary secretion of men on ADT. The sebaceous glands of the skin are known to contain the enzyme systems required for local conversion of circulating weaker androgens to potent forms and also for direct synthesis of some androgens from cholesterol. Furthermore, skin is one of the extragonadal tissues that express LH receptors and thus LHRHa treatment may potentially alter the axillary steroid production [36, 37]. However, our results showed no change in the cutaneous secretion of major androgens including $\mathrm{T}$ and DHT following ADT. The levels of metabolic intermediates androsterone and $5 \alpha$ androstane-3,17-dione in the axillary secretions were significantly lowered from the baseline. LHRHa-induced suppression of de novo cutaneous production of $\mathrm{T}$ and DHT may have caused shunting of the circulating weaker androgenic metabolites androsterone and $5 \alpha$-androstane-3,17-dione toward the synthesis of T and DHT, resulting in the decreased level of the former in axillary secretions and maintenance of the levels of the latter.

Some androstenes have been associated with chemosignal- 
T A B L E 1. Estimated marginal mean ( \pm S.E.M) odor ratings for two odor samples (at baseline and three months later) using repeated measures ANOVA.

\begin{tabular}{|c|c|c|c|c|c|c|}
\hline \multirow{2}{*}{ Raters } & \multirow{2}{*}{ Rating } & \multicolumn{2}{|c|}{ Control group $(\mathrm{n}=10)$} & \multicolumn{2}{|c|}{ Study group $(\mathrm{n}=10)$} & \multirow{2}{*}{$P$} \\
\hline & & Baseline & 3 months & Baseline & 3 months & \\
\hline \multirow{3}{*}{ Male } & Attractiveness & $2.92 \pm 0.18$ & $2.92 \pm 0.14$ & $2.72 \pm 0.18$ & $2.83 \pm 0.14$ & 0.64 \\
\hline & Masculinity & $3.13 \pm 0.13$ & $2.79 \pm 0.14$ & $3.27 \pm 0.13$ & $3.22 \pm 0.14$ & 0.30 \\
\hline & Intensity & $3.27 \pm 0.28$ & $2.61 \pm 0.18$ & $3.17 \pm 0.28$ & $3.02 \pm 0.18$ & 0.28 \\
\hline \multirow{3}{*}{ Female } & Attractiveness & $2.68 \pm 0.18$ & $2.73 \pm 0.23$ & $2.55 \pm 0.18$ & $2.61 \pm 0.23$ & 0.95 \\
\hline & Masculinity & $2.94 \pm 0.16$ & $2.82 \pm 0.21$ & $2.86 \pm 0.16$ & $2.75 \pm 0.21$ & 0.98 \\
\hline & Intensity & $3.03 \pm 0.27$ & $2.73 \pm 0.19$ & $2.88 \pm 0.27$ & $2.64+0.19$ & 0.89 \\
\hline \multirow{3}{*}{ Combined } & Attractiveness & $2.81 \pm 0.15$ & $2.83 \pm 0.15$ & $2.65 \pm 0.15$ & $2.73 \pm 0.15$ & 0.82 \\
\hline & Masculinity & $3.04 \pm 0.09$ & $2.80 \pm 0.12$ & $3.06 \pm 0.09$ & $2.98 \pm 0.12$ & 0.38 \\
\hline & Intensity & $3.15 \pm 0.24$ & $2.68 \pm 0.15$ & $3.03 \pm 0.24$ & $2.83 \pm 0.15$ & 0.50 \\
\hline
\end{tabular}

T A B L E 2. Comparison of steroid concentration in odor samples of the study group (at baseline and three months later) using Wilcoxon Signed Rank Test.

\begin{tabular}{lccc}
\hline \multirow{2}{*}{ Steroids } & Baseline $(\mathrm{ng} / \mathrm{mL})$ & 3 months $(\mathrm{ng} / \mathrm{mL})$ & $P$ \\
\cline { 2 - 4 } & Median $(\mathrm{Q} 1, \mathrm{Q} 3)$ & Median $(\mathrm{Q} 1, \mathrm{Q} 3)$ & $0.02^{*}$ \\
\hline Androsterone & $8.97(4.36,12.82)$ & $2.24(1.67,4.07)$ & $0.02^{*}$ \\
$5 \alpha$-Androstane-3,17-dione & $8.44(5.88,32.21)$ & $5.75(2.04,12.09)$ & 0.11 \\
5-Pregnan-17-ol-3,20-dione & $0.67(0.39,3.81)$ & $1(0.66,6.94)$ & 0.16 \\
Dehydroepiandrosterone & $1343.05(473.09,2272.63)$ & $736.4(362.03,1742.95)$ & 0.22 \\
Dihydrotestosterone & $3.67(2.64,6.22)$ & $2.31(0.93,4.28)$ & 0.30 \\
4-Pregnen-17-ol-3,20-dione & $1.24(0.8,2.13)$ & $1.22(0.7,1.47)$ & 0.30 \\
Pregnenolone & $798.67(623.89,1051.02)$ & $756.97(565.75,973.12)$ & 0.47 \\
Pregnan-3,20-dione & $32.38(23.83,35.96)$ & $27.41(22.9,34.07)$ & 0.47 \\
17-OH Pregnenolone & $21.81(18.66,29.64)$ & $19.21(16.45,25.89)$ & 0.58 \\
Cortisol & $39.06(13.93,96.64)$ & $26.44(10.25,46.99)$ & 0.81 \\
5-Pregnan-3-ol-20-dione & $7.36(6.6,8.62)$ & $7.42(6.83,8.76)$ & 0.94 \\
Testosterone & $10.97(9.44,13.22)$ & $9.86(8.76,13.86)$ & 0.94 \\
4-Pregnan-3,17-diol-20-one & $7.19(4.07,42.71)$ & $12.55(2.93,49.65)$ & 0.94 \\
Epi-Testosterone & $16.49(13.76,18.3)$ & $16.76(13.27,19.32)$ & 1.00 \\
Androstenedione & $12.75(9.17,30.53)$ & $10.42(9.24,15.74)$ & 1.00 \\
Progestesterone & $37.38(27.95,46.47)$ & $31.04(23.83,40.83)$ & \\
\hline *Difference is considered significant $2<P(0.05$. & & \\
\end{tabular}

*Difference is considered significant at $P<0.05$.

ing in our species [19]. On average, the men on an LHRHa had a decrease in their androsterone and $5 \alpha$-androstane3,17-dione cutaneous secretion to nearly half of the average amount found in eugonadal men. As such, the LC-MS data suggest that the decreased concentration of androsterone and $5 \alpha$-androstane-3,17-dione in the axillary secretions of the men on ADT might show up in the olfactory assessment of the shirts by the panel of sniffers. It is surprising then that the assessment of the scent emitted from the shirts on attractiveness, masculinity and intensity all yielded negative results (Table 1). We are left to speculate why.

While our data failed to show discernible shifts in axillary odor after 3 months of LHRHa therapy, there are several explanations worthy of further investigation. In addition to androsterone and $5 \alpha$-androstane-3,17-dione, axillary secretions contain a wide array of odorant molecules including cholesterol, cholesteryl esters, unsaturated/hydroxylated branched fatty acids and sulfanylalkanols [38], which are not necessarily downregulated by ADT. All metabolites that are lipid soluble can be stored in body fat, including the subcutaneous fat proximal to axillary apocrine glands. Those molecules may be retained in the fat longer than the three months the participants were on ADT. This suggests that we might have found a significant difference in perceived body odor, if there were a longer time period between the baseline and final measurements. Our results provide evidence though that suppressing serum $\mathrm{T}$ concentrations may not significantly influence perceived odor quality in the short time period of our study.

Humans have been shown to discriminate between age based on emitted body odor and that this effect was mediated mainly by the scent of older age groups [23]. Earlier work using gas-chromatography mass spectrometry enabled detection of 2-Nonenal, a strongly odoriferous aldehyde derived from oxidative degradation of fatty acids, but only in individuals 40 years or older, and the quantity was shown to increase with advancing age [39]. In our study, odor samples were provided by elderly men both with and without androgen suppression. Changes in the odor perceived by the sniffers of the shirts from the PC patients before and after being treated with an LHRHa may have been masked by other odor constituents, such as 2-Nonenal. 
The age difference between the men, who wore the shirts, and the panel, who smelled the shirts, may also have influenced the results. Moreover, there is great diversity in human olfaction, which is known to be mediated mainly by genotypic variations [40]. The reliability of ratings may have been compromised due to such variability in raters' individual olfactory ability [41], although relatively large numbers of raters were employed to minimize this effect. In addition to this, the sampling technique employed involved participant's wearing T-shirts in bed for two consecutive nights and following the instructions about dietary restrictions and hygiene. There was no objective way to determine participant's adherence to the instructions, and this may have affected the outcome.

We recruited and subsequently collected shirt samples across all seasons. Anecdotal observations suggest seasonal variation in axillary sweat production. Personal grooming habits including shaving of axillary hair have also been shown to affect axillary odor [42]. The cutaneous microflora of individuals is unique in terms of its diversity, composition and microbial load and the action of cutaneous microflora on constituents of axillary sweat has been shown to alter odor characteristics $[43,44]$. Our sampling method did not control for any of those factors.

Because of potential inadequacies of the sampling methods, plus the subjective assessments of odor in our study, the issue of whether gonadal hormone suppression in men affects their olfactory social signaling remains unresolved. Given our small sample size, our research can best be viewed as a pilot study confirming the feasibility of the methodologies for analyzing the impact of gonadal hormone suppression on steroid cutaneous secretion.

The social implications of body odor for our species are undeniable. As such, the psychological and behavioral implications of endocrine treatments warrant further study not only in PC patients, but in other populations subjected to gonadal hormone manipulations. The literature, for example, exploring the impact of visible changes in body form from breast cancer surgeries on self-esteem and intimate relationship is enormous [45]. In contrast the potential implications of endocrine treatments on body odor for breast cancer patients has received little or no research.

A growing concern relates to the use of puberty blocking agents for pubescent individuals with gender dysphoria [4648]. There are no data that we know of on the long-term implications on socially significant olfactory signals from individuals exposed to LHRHa at the time that they would normally go through puberty. This concern extends as well to transgenders undergoing GAT $[49,50]$. In a recent study by Kranz et al. [51] in transgender individuals, no significant correlation was reported between sex hormones and odor perception after four months of GAT, but the study employed sniffing sticks which did not include body odor. However, the fact that LHRHa drugs change the steroid secretion of PC patients raises a cautionary flag that they may also change in the skin secretions in individuals treated for gender dysphoria. Whether they cause such changes and whether they influence olfactory social signaling remains to be explored. In the interim, hormonal treatments labelled as "gender affirming", may not provide complete gender affirmation if they do not produce a gender specific olfactory signal.

\section{Conclusions}

Our study confirms that three months of ADT can significantly alter axillary secretion of some steroids. We found some evidence of a general depression of androgens in the secretions, but this was only statistically significant for the androstenes, androsterone and $5 \alpha$-androstane-3,17-dione. We anticipated that such changes in secreted hormones would alter the perceived odor of the men's shirts. This was not found, and various factors may account for this negative result. Future research on chemosignaling from men on LHRHa may need to use a more age matched panel of sniffers and the men may need to be androgen-suppressed for a longer time. It remains an open question as to whether there are olfactory shifts associated with LHRHa drugs that influence the social and sexual relations of men treated with LHRHa for PC and other populations on these same pharmaceutical agents.

\section{Abbreviations}

ADT, androgen deprivation therapy; CYP17, cytochrome P450 $17 \alpha$ hydroxylase/17,20 lyase (CYP17); d3DHT, dihydrotestosterone; d3T, deuterated testosterone; E2, estradiol; EtOAc, ethyl acetate; GAT, gender-affirming therapy; $\mathrm{HA} / \mathrm{MeOH}$, hydroxylamine/methanol; IS, internal standard; LC-MS, liquid chromatography-mass spectrometry; LH, luteinizing hormone; LHRH, luteinizing hormone-releasing hormone; LHRHa, luteinizing hormonereleasing hormone agonists; PC, prostate cancer; $\mathrm{T}$, testosterone.

\section{Author contributions}

SIAS, HCPW, PDA, RJW and SCR contributed to the concept, design, definition of intellectual content, manuscript preparation, manuscript editing and manuscript review. SIAS, SCR, VT, JTF and CA performed the olfactory assessment and analyzed the olfactory data. HHA and ESTG performed the chemical assessment and RHB analyzed the chemical data.

\section{Ethics approval and consent to participate}

All samples used in the study were collected after approval as an Imperial College Healthcare Tissue Bank (ICHTB) subcollection (Ref. No. Sur_PA_12_044) and were later issued for analysis following approval (Project No. R14110). ICHTB is licensed by Human Tissue Authority (No. 12275) and funded by the tissue banking theme of National Institute of Health Research and Imperial Biomedical Research Centre. ICHTB is authorized by the Wales National Research Ethics Service (12/WA/0196) to release human material for 
research.

\section{Acknowledgment}

We are thankful to Steven Mangar for aiding in recruitment for the study and Richard Harvey for helping with storage of study samples.

\section{Funding}

This work was part of the doctoral study of SIAS supported by the Commonwealth Scholarship Commission (PKCA-201185). VT and JTF were supported by the Ministry of Education, Youth, and Sports NPU I program (No. LO1611) and by the Czech Science Foundation (Grant No. P407/21-29772S).

\section{Conflict of in terest}

The authors declare no conflict of interest.

\section{References}

[1] Thomas BC, Neal DE. Androgen deprivation treatment in prostate cancer. British Medical Journal. 2013; 346: e8555.

[2] Ahmadi H, Daneshmand S. Androgen deprivation therapy for prostate cancer: long-term safety and patient outcomes. Patient Related Outcome Measures. 2014; 5: 63-70.

[3] Bangalore Krishna K, Fuqua JS, Rogol A, Klein K, Popovic J, Houk C, et al. Use of gonadotropin-releasing hormone analogs in children: update by an international consortium. Hormone Research in Paediatrics. 2019; 91: 357-372.

[4] Shah SIA. Emerging potential of parenteral estrogen as androgen deprivation therapy for prostate cancer. South Asian Journal of Cancer. 2015; 4: 95-97.

[5] Sharifi N, Gulley JL, Dahut WL. Androgen deprivation therapy for prostate cancer. Journal of the American Medical Association. 2005; 294: 239-244.

[6] Freedland SJ, Eastham J, Shore N. Androgen deprivation therapy and estrogen deficiency induced adverse effects in the treatment of prostate cancer. Prostate Cancer and Prostatic Diseases. 2009; 12: 333-338.

[7] Donovan KA, Walker LM, Wassersug RJ, Thompson LMA, Robinson JW. Psychological effects of androgen-deprivation therapy on men with prostate cancer and their partners. Cancer. 2015; 121: 4286-4299.

[8] Tsang VWL, Skead C, Wassersug RJ, Palmer-Hague JL. Impact of prostate cancer treatments on men's understanding of their masculinity. Psychology of Men \& Masculinities. 2019; 20: 214-225.

[9] Eisenegger C, Haushofer J, Fehr E. The role of testosterone in social interaction. Trends in Cognitive Sciences. 2011; 15: 263-271.

[10] Walker LM, Santos-Iglesias P, Robinson J. Mood, sexuality, and relational intimacy after starting androgen deprivation therapy: implications for couples. Supportive Care in Cancer. 2018; 26: 38353842.

[11] Wassersug RJ. Maintaining intimacy for prostate cancer patients on androgen deprivation therapy. Current Opinion in Supportive and Palliative Care. 2016; 10: 55-65.

[12] Elliott S, Latini DM, Walker LM, Wassersug R, Robinson JW, ADT Survivorship Working Group. Androgen deprivation therapy for prostate cancer: recommendations to improve patient and partner quality of life. The Journal of Sexual Medicine. 2010; 7: 2996-3010.

[13] Navon L, Morag A. Advanced prostate cancer patients' relationships with their spouses following hormonal therapy. European Journal of Oncology Nursing. 2003; 7: 73-80.

[14] Wyatt TD. The search for human pheromones: the lost decades and the necessity of returning to first principles. Proceedings. Biological Sciences. 2015; 282: 20142994.
[15] Gosling LM, Roberts SC. Scent-marking by male mammals: cheatproof signals to competitors and mates. Advances in the Study of Behavior. 2001; 30: 169-217.

[16] Dandine-Roulland C, Laurent R, Dall'Ara I, Toupance B, Chaix R. Genomic evidence for MHC disassortative mating in humans. Proceedings of the Royal Society B: Biological Sciences. 2019; 286: 20182664.

[17] Havlíček J, Fialová J, Roberts SC. Individual variation in body odor. Springer Handbook of Odor. 2017; 28: 125-126.

[18] Prokop-Prigge KA, Greene K, Varallo L, Wysocki CJ, Preti G. The effect of ethnicity on human axillary odorant production. Journal of Chemical Ecology. 2016; 42: 33-39.

[19] Havlicek J, Murray AK, Saxton TK, Roberts SC. Current issues in the study of androstenes in human chemosignaling. Vitamins and Hormones. 2010; 83: 47-81.

[20] Saxton TK, Lyndon A, Little AC, Roberts SC. Evidence that androstadienone, a putative human chemosignal, modulates women's attributions of men's attractiveness. Hormones and Behavior. 2008; 54 : 597-601.

[21] Havlicek J, Roberts SC, Flegr J. Women's preference for dominant male odour: effects of menstrual cycle and relationship status. Biology Letters. 2005; 1: 256-259.

[22] Thornhill R, Chapman JF, Gangestad SW. Women's preferences for men's scents associated with testosterone and cortisol levels: Patterns across the ovulatory cycle. Evolution and Human Behavior. 2013; 34: 216-221.

[23] Mitro S, Gordon AR, Olsson MJ, Lundström JN. The smell of age: perception and discrimination of body odors of different ages. PLoS ONE. 2012; 7: e38110.

[24] Lobmaier JS, Fischbacher U, Wirthmüller U, Knoch D. The scent of attractiveness: levels of reproductive hormones explain individual differences in women's body odour. Proceedings of the Royal Society B: Biological Sciences. 2018; 285: 20181520

[25] Jacob S, Hayreh DJ, McClintock MK. Context-dependent effects of steroid chemosignals on human physiology and mood. Physiology \& Behavior. 2001; 74: 15-27.

[26] Berglund H, Lindström P, Savic I. Brain response to putative pheromones in lesbian women. Proceedings of the National Academy of Sciences of the United States of America. 2006; 103: 8269-8274.

[27] Verhaeghe J, Gheysen R, Enzlin P. Pheromones and their effect on women's mood and sexuality. Facts, Views \& Vision in ObGyn. 2013; 5: 189-195.

[28] Preti G, Wysocki CJ, Barnhart KT, Sondheimer SJ, Leyden JJ. Male axillary extracts contain pheromones that affect pulsatile secretion of luteinizing hormone and mood in women recipients. Biology of Reproduction. 2003; 68: 2107-2113.

[29] d'Ettorre P, Bueno S, Rödel HG, Megherbi H, Seigneuric A, Schaal B, et al. Exposure to androstenes influences processing of emotional words. Frontiers in Ecology and Evolution. 2018; 5: 169.

[30] Havlíček J, Lenochová P, Oberzaucher E, Grammer K, Roberts SC. Does length of sampling affect quality of body odor samples? Chemosensory Perception. 2011; 4: 186-194.

[31] Roberts SC, Gosling LM, Carter V, Petrie M. MHC-correlated odour preferences in humans and the use of oral contraceptives. Proceedings: Biological Sciences. 2008; 275: 2715-2722.

[32] Lenochova P, Roberts SC, Havlicek J. Methods of human body odor sampling: the effect of freezing. Chemical Senses. 2009; 34: 127-138.

[33] Martinec Nováková LM, Havlíček J, Roberts SC. Olfactory processing and odor specificity: a meta-analysis of menstrual cycle variation in olfactory sensitivity. Anthropological Review. 2014; 77: 331-345.

[34] Gordon JA, Noble JW, Midha A, Derakhshan F, Wang G, Adomat HH, et al. Upregulation of scavenger receptor B1 is required for steroidogenic and nonsteroidogenic cholesterol metabolism in prostate cancer. Cancer Research. 2019; 79: 3320-3331.

[35] Deb S, Pham S, Ming D, Chin MY, Adomat H, Hurtado-Coll A, et al. Characterization of precursor-dependent steroidogenesis in human prostate cancer models. Cancers. 2018; 10: 343.

[36] Chen W, Thiboutot D, Zouboulis CC. Cutaneous androgen 
metabolism: basic research and clinical perspectives. The Journal of Investigative Dermatology. 2002; 119: 992-1007.

[37] Bird J, Li X, Lei ZM, Sanfilippo J, Yussman MA, Rao CV. Luteinizing hormone and human chorionic gonadotropin decrease type $25 \alpha$ reductase and androgen receptor protein levels in women's skin. The Journal of Clinical Endocrinology \& Metabolism. 1998; 83: 1776-1782.

[38] Pandey SK, Kim K. Human body-odor components and their determination. TrAC Trends in Analytical Chemistry. 2011; 30: 784-796.

[39] Haze S, Gozu Y, Nakamura S, Kohno Y, Sawano K, Ohta H, et al. 2-Nonenal newly found in human body odor tends to increase with aging. The Journal of Investigative Dermatology. 2001; 116: 520-524.

[40] Zhang X, Firestein S. Nose thyself: individuality in the human olfactory genome. Genome Biology. 2007; 8: 230.

[41] Hasin-Brumshtein Y, Lancet D, Olender T. Human olfaction: from genomic variation to phenotypic diversity. Trends in Genetics. 2009; 25: $178-184$

[42] Kohoutová D, Rubešová $A$, Havlíček J. Shaving of axillary hair has only a transient effect on perceived body odor pleasantness. Behavioral Ecology and Sociobiology. 2012; 66: 569-581.

[43] Xu Y, Dixon SJ, Brereton RG, Soini HA, Novotny MV, Trebesius $\mathrm{K}$, et al. Comparison of human axillary odour profiles obtained by gas chromatography/mass spectrometry and skin microbial profiles obtained by denaturing gradient gel electrophoresis using multivariate pattern recognition. Metabolomics. 2007; 3: 427-437.
[44] Sterndorff EB, Russel J, Jakobsen J, Mortensen MS, Gori K, Herschend $\mathrm{J}$, et al. The T-shirt microbiome is distinct between individuals and shaped by washing and fabric type. Environmental Research. 2020; 185: 109449.

[45] Rumsey N, Harcourt D. Oxford handbook of the psychology of appearance. Oxford University Press. New York, NY, US. 2012.

[46] Unger CA. Hormone therapy for transgender patients. Translational Andrology and Urology. 2016; 5: 877-884.

[47] Mahfouda S, Moore JK, Siafarikas A, Zepf FD, Lin A. Puberty suppression in transgender children and adolescents. The Lancet Diabetes \& Endocrinology. 2017; 5: 816-826.

[48] Anacker C, Sydnor E, Chen BK, LaGamma CC, McGowan JC, Mastrodonato A, et al. Behavioral and neurobiological effects of GnRH agonist treatment in mice-potential implications for puberty suppression in transgender individuals. Neuropsychopharmacology. 2021; 46: 882-890.

[49] Frey JD, Poudrier G, Thomson JE, Hazen A. A Historical review of gender-affirming medicine: focus on genital reconstruction surgery. The Journal of Sexual Medicine. 2017; 14: 991-1002.

[50] Radix A. Hormone therapy for transgender adults. The Urologic Clinics of North America. 2019; 46: 467-473.

[51] Kranz GS, Kaufmann U, Lanzenberger R. Probing the impact of gender-affirming hormone treatment on odor perception. Chemical Senses. 2020; 45: 37-44. 